\title{
Observations on the physical basis of mechanical behaviour
}

\author{
S RAMASESHAN \\ Indian Institute of Science, Bangalore 560012 , India
}

\section{General remarks}

It gives me great pleasure to participate in this Discussion Meeting. This is perhaps the first time that physicists, materials scientists and metallurgists in India have planned a meeting to understand each other and to see whether phenomena not completely comprehended by one group can be looked into by another or jointly. The motivations are so praiseworthy that I am sure something will come out of such meetings. If these meetings are to be successful many obstacles have to be surmounted. The first, of course, is that of language. Each person here must understand the jargon of the other. The second is to make a conscious attempt to change one's attitudes.

The metallurgists and physicists today appear to be two different animals. The purpose of this meeting is to see that the barriers that have been artificially raised are removed. One has only to read the fascinating books by Cyril Stanley Smith, From Art to Science and $A$ Search for Structure to realise that metallurgy which has been an empirical science for a long time has been eminently successful; and yet what is common to the village blacksmith is very mysterious to the physicist. Science was injected into metallurgy only recently. Most metallurgists, like chemists, are pragmatists. They reduce many sophisticated theories to thumb rules which work and which have advanced the fields most spectacularly. However, there is a tendency among many of them not to go too deeply into the fundamentals of the phenomena they observe. The physicist, on the other hand, choose rather simple ideal systems and model them. As this approach has been rather successful in physics he feels that by using a similar one he can "clean" up many of the problems which the metallurgist does not understand. In this connection, I must recall what Prof. Andrew Huxley-a physicist himself who entered biology and distinguished himself - said when he lectured at the Indian Institute of Science, Bangalore on Physical Sciences and Biology. Biology, according to him, is inherently complex. Simplistic models sometimes hinder rather than advance the subject. It is the complexity and the basis of this complexity that one must try to understand and describe. The physicist, if he is to be useful at all, must enter biology with great humility, with a genuine intent to understand the subject and its spirit. What Huxley said of biology is, in my view, applicable to metallurgy and materials science.

In each field of science when sufficient knowledge accumulates, a great step is usually taken which throws new light and opens up new vistas. This happened to biology about 25 years ago when biologists and crystallographers combined to unravel the structure of DNA and caused a revolution which biology is still experiencing.

For metallurgy there was one such great flash in 1934 when the theory of dislocation was put forward by physicists which opened the gates for the understanding of the mechanism of strengthening, crystal growth etc. Fifty years have now passed and one 
feels that a coming together of scientists in related fields may possibly help is taking another important step even if it is not as revolutionary as the dislocation theory. Being an experimenter myself I must stress the importance of experiments. Unfortunately the experimental approach is sometimes neglected by physicists in India. Experiment forms the backbone of progress is any field. I am told that Prof. Mott often stressed that every theoretical paper or idea must suggest a clean interesting experiment which can be performed. I do hope that at the end of this meetin $\tilde{\varepsilon}^{-}$crucial experiments will be suggested which will help to bring about the proper understanding of some of the puzzling phenomena in this field.

If such meetings are held often they would create a community of scientists interested in mechanical behaviour of materials. They may also provoke individuals or groups with complementary competences to collaborate. One hopes that the theorists and experimenters would join hands to look into some of the exciting problems in this field. Following the spirit advocated by the organisers of this Meeting, in this inaugural talk I shall mention a few experiments which I and my group have performed (many of which are unpublished) and others which are not so well-known, that have come my way. I shall also raise some questions which have troubled me particularly those connected with crack generation, hardness and its variation, friction, wear, surface states and the nature of grain boundaries.*

\section{Welding of surfaces and generation of cracks}

In some experiments done in 1944 I noticed that diamond surfaces freshly cleaved in vacuum $\left(10^{-5}\right.$ torr) do not rejoin when brought together almost immediately after cleaving. It was also possible to show by studying the Jamin effect (i.e. by ellipsometric methods) that a thin film is formed on the cleaved surface. In 1948 Gwathemay showed that cleaved copper single crystal welded together in vacuum $\left(10^{-8}\right.$ torr $)$ a phenomenon now common in space.**

At the surface of a metal due to discontinuity there would be a spill-over of electrons and the consequent charge imbalance is prevented by the electrons collectively conspiring to set themselves into oscillations. When two clean metal surfaces are brought close to each other these oscillations in each piece (which are independent) can become coupled and they go backwards and forwards from one piece to the other, bonding the two surfaces. Another crucial question is: Can stress concentration in a metal cause an instability in the electron oscillations so that decoupling can take place causing the formation of two surfaces (i.e. a crack?) These are topics of great interest worthy of some thought and discussion.***

\footnotetext{
* No attempt is made to pad it up to make it more intelligible!

** I am not aware of any experiments done in space attempting to join or weld diamonds. It is not clear to me why diamond surfaces should not also weld as copper surfaces do. The dangling bonds in diamond may help to chemisorb atoms to form a thin film. In metals also thin films are formed by physical adsorption. One wonders whether the difference in the magnitude of the energies of chemisorption and physisorption is the real cause of the two types of behaviour.

*** Somo may disagree with the conjecture that two metal surfaces weld together in vacuum because the surface plasmons go backwards and forwards from one piece to another. It is strange that the magnitude of the surface energy is very close to the contribution of the correlation part of the electron energy. Does this imply that for some reason there is a cancellation of some of the very large terms like the lattice contribution, the kinetic energy of the electron etc? Similarly, when one considers the cohesive energy (next section) is there also a cancellation of the Madelung term, corresponding to the kisetic energy of the electron so that the electrons are mostly responsible for the cohesive energy.
} 
Crude measurements (by us) of the stress optic coefficients of metals indicate that stress has considerable effect on the electrons. Stress distorts the lattice and due to this there are two optical effects. Firstly, the anisotropic distribution of the polarisable atoms in the strained lattice alters the index $(n)$ and absorption $(k)$ tensors. Secondly, electrons moving in the strained lattice can also alter the $n$ and $k$ tensors. The latter effect appears to be quite large and is opposite to that of the former.

\section{Do electrons contribute to the hardness of metals?}

Since electrons do contribute so much to the cohesive energy of a solid, it seems very probable (at least to me) they contribute to the hardness of a metal. $\dagger$

Some compounds like rare earth chalcogenides (example $\mathrm{SmS}$ which has the $\mathrm{NaCl}$ structure) are semiconductors and become metals under pressure, undergoing isostructural semiconductor-to-metal transformation. There is a volume change and the resistancc drops by five to six orders of magnitudes. These are peculiar metals which have positive and negative ions inside with "free electrons" in them. By measuring the hardness of the two phases one can get an idea as to whether electrons do contribute to hardness or not. To make measurements at high pressures is not easy although the method mentioned in the next section could be adopted for this purpose. The effect of the density change on hardness must also be taken into account.

A similar isostructural semiconductor-metal-transition can be effected by adding adequate amounts of rare earth metal which simulates the effects of pressure (e.g. SmS is a semiconductor while $\mathrm{Sm}_{0.85}, \mathrm{Gd}_{0.15} \mathrm{~S}$ is a metal). The hardness of the metal is much higher than that of the semiconductor. But one cannot be certain whether this increase is due to the electrons, the change of density or the additional Gd atoms in the substance.

\section{The hardness pendulum - The measurement of the variations of hardness}

The hardness pendulum, devised by experimentors in USSR, in which a loaded cylinder oscillates on the surface under study can be used to measure hardness of the metal under a variety of conditions. The hardness is estimated from the rate of damping of the oscillations. Liquids can be placed between the surface and the cylinder to study the effects of adsorption and hardness. With an electrolyte, variation of hardness can be studied with varying potentials applied on the system. The hardness of the material increases with increasing potential and becomes maximum when the charge on the surface is zero and it decreases as the surface becomes progressively negatively charged. In fact, this curve is very similar to the variation of the interfacial tension of $\mathrm{Hg}$ solution interface with applied voltage indicating clearly that the surface energy (which changes with the application of potential) is involved in the phenomenon of hardness. By

\footnotetext{
† I am not at all happy using the word 'electron' in this loose manner as I am aware that there are several contributions to the energy from electrons themselves and they even have opposite signs.

$¥$ To my mind the question "do electrons" contribute to the hardness of metals is an important one and deserves further theoretical and experimental investigation.
} 
analogy with the $\mathrm{Hg}$ solution interface the effect of atoms absorbed on the surface was studied. Many patents on additives to increase the machining rates have resulted from this work.

It is also found that the effect of absorption of neutral atoms decreases the hardness. It is also a known fact that the work function $W(\phi)$ of a refractory transition metal is lowered by as much as $4 \mathrm{eV}$ by a monolayer of Cs. This is possibly the reason why adsorption can decrease hardness. Theoretical calculations of the electron distribution on the surface have also been done. When a tin crystal is coated with liquid gallium the crystal becomes brittle. Laue photographs show that a single crystal becomes polycrystalline. It is thought that the grains are held together by surface tension forces of the liquid. The strength of the solid decreases considerably although its ductility is not affected.

\section{Friction, surface roughness and surface states}

The second law of friction discovered by the great Leonardo da Vinci (which states that the coefficient of friction $(\mu)$ is independent of the surface area) appears mysterious to many when they first come to know of it. To explain this experimental law one must seek a phenomenon by which a true area of contact is proportional to load (the first law states that the $\mu \propto$ load). The real surface of any material consists of microscopic hills and valleys (asperities). When two surfaces touch, the area of contact being small the stress is very large. An elastic deformation takes place first and this is followed by a plastic one till the total area of contact increases to a value which can support the load. This phenomenon can be elegantly demonstrated by observing intensively the total internal reflection in a right angle prism. When the surface (with its asperities) is laid on the hypotenuse of the prism wherever there is a real contact there is no total internal reflection, and where there is no contact, evanescent wave returns into the medium of the prism. The intensity of the totally reflected beam decreases with load.

The existence of the asperities has also been demonstrated by measurements of the AC surface resistance (at very high frequencies $24000 \mathrm{MHz}$ when the skin depth is as low as $0.4 \mu$ ) and this surface resistance is much greater than the DC resistance. If the surface is bathed in carbon monoxide, the AC resistance increases considerably indicating that the electrons are trapped in the surface states created by $\mathrm{CO}$.

\section{Electrons and metals}

There are many other questions which require consideration. What is the submicroscopic beilby layer which forms when one metal is rubbed against another? And which spalls off during wear producing hard particles? Why is it harder and more brittle than the parent metal-although it is said to have the same composition? Has the metal turned amorphous due to the high temperatures and pressures produced during wear? Does the contribution of the electron to cohesive energy increase in amorphous metals, making them harder and corrosive resistant? Electron micrographs definitely indicate that the boundaries in large angle polycrystalline metals and alloys are extremely thin (about 3 to 5 atomic layers). Are these really "amorphous" regions with coordination polyhydra postulated by Bernal for "liquids"? Are polycrystals stronger than the monocrystals because dislocation cannot wander across the grain boundaries or is it 
because electron "lattice" interactions at the grain boundaries contribute to the cohesive energy?

We know that the dislocation theory has been very successful but are we leaning a bit too heavily on it, relegating the effect of free electrons on the mechanical properties, to the background?

Soon after the discovery of the quantum theory, it was appreciated that the physical basis for the optical and magnetic properties of solids must be found at the microscopic level. Unfortunately, the physical basis of mechanical behaviour was relegated as engineering. Only some outstanding scientists like Mott and Pierls appreciated that one must look at microscopic phenomena to understand mechanical behaviour also. Soon after the advent of the dislocation theory, their interest shifted to other problems and physicists have been very reluctant to contribute to this complex but important field. It is a bit ironical because the most dramatic and obvious property of a solid is, of course, its mechanical strength. This meeting clearly shows that we in India have a unique opportunity to make some important contributions before the rest of the world wakes up, so to say. 